\title{
External sources of RFI at the GMRT: Methods for control and co-existence with commercial users
}

\author{
Pravin Ashok Raybole ${ }^{1}$ \\ GMRT-NCRA-TIFR \\ P.O Box No. 6, Narayangon, Pune, India. \\ E-mail: pravinegmrt.ncra.tifr.res.in
}

\section{S. Sureshkumar}

GMRT-NCRA-TIFR

P.O.Box No. 6, Narayangaon, Pune, India.

E-mail: skumar@ncra.tifr.res.in

The Giant Meterwave Radio Telescope (GMRT) observatory in India consists of 30 antennas in a Y-shaped array, spread out over a $14 \mathrm{~km}$ radius from the central processing center. This makes the protection of radio telescopes from increasing radio frequency interference (RFI) at meter wavelengths quite challenging. Radio telescopes are built with broadband receivers to observe beyond the narrow allocated radio astronomy bands, so protecting the receivers from out-ofband emission is a major task. With the rapid increase in mobile communication, TV and FM Radio transmitters, police wireless, industries and power line distribution networks, private and public transport, there is a significant increase in RFI at low frequencies.

This paper presents the results of above studies of these external sources of RFI, their effect on the receiver system, and of various methods adopted to minimize the same to protect the GMRT. This paper also describes various control experiments carried out, with measurements made over the past 10 years, and methods adopted to co-exist with commercial communication operators and industries to control the RFI environment at the GMRT

The GMRT RFI team has been able to detect and correct 1800 radiating domestic TV boosters spread over a $20 \mathrm{~km}$ radius with a simple low cost solution. Using RF and Ultrasonic detectors, dense electrical distribution networks with LT and HT power distribution lines and single phase, 3 phase transformer installations in the neighbor hood are monitored, and steps have been taken to control various power line sources of interference that have affected observations. The GMRT also faces challenges from many GSM and CDMA mobile operators in the region around the GMRT. We have been able to negotiate for a change in operating band and in the orientation of cell towers etc. to protect the GMRT. Efforts are also ongoing to monitor and control the interference from industries operating in nearby regions as well as from internally generated RFI at the GMRT.

RFI mitigation workshop Groningen, the Netherlands March 29-31, 2010

Pravin Ashok Raybole 


\section{Introduction}

Radio telescopes built with wide band receivers that operate well beyond the allocated radio astronomy bands need protection from ever increasing radio frequency interference. The GMRT is one such instrument that tries to provide seamless coverage from $50 \mathrm{MHz}$ to $1450 \mathrm{MHz}$ which uses the available free RF bands for radio astronomy purposes. Such radio telescopes have very high dynamic ranges to accommodate the large increase in power when RFI occurs. This is, however, not always possible, so efforts to control these sources and ameliorate sources RFI are required. At the GMRT, in addition to protecting the existing radio astronomy bands, efforts are being made to reduce radio frequency interference from sources such as mobile, TV \& FM radio transmitters, police wireless, industry, power line interference from the electric company, and from private and public transport.

\subsection{RFI Environment at the GMRT}

Radio frequency interference to the GMRT is classified into two broad categories, (i) intentional radiation, and (ii) unintentional radiation. Intentional radiations comes from known standard commercial transmitters of FM radio, TV, Satellites, air traffic control, police wireless, and cell phone towers. Unintentional radiations includes interference from faulty power lines, saturated or oscillating TV boosters, un-terminated cable TV networks, industries, welding machines, vehicles, instruments, and computer \& networking devices. With technological advances and an increase in living standards in the region around GMRT, it is necessary to monitor these radiating sources and their interference power levels from the neighborhood of the GMRT. The spectrum analyzer plots showing the standard commercial operators around the GMRT can be seen in figure $1.1 \mathrm{a}, 1.1 \mathrm{~b}$ and $1.1 \mathrm{c}$.

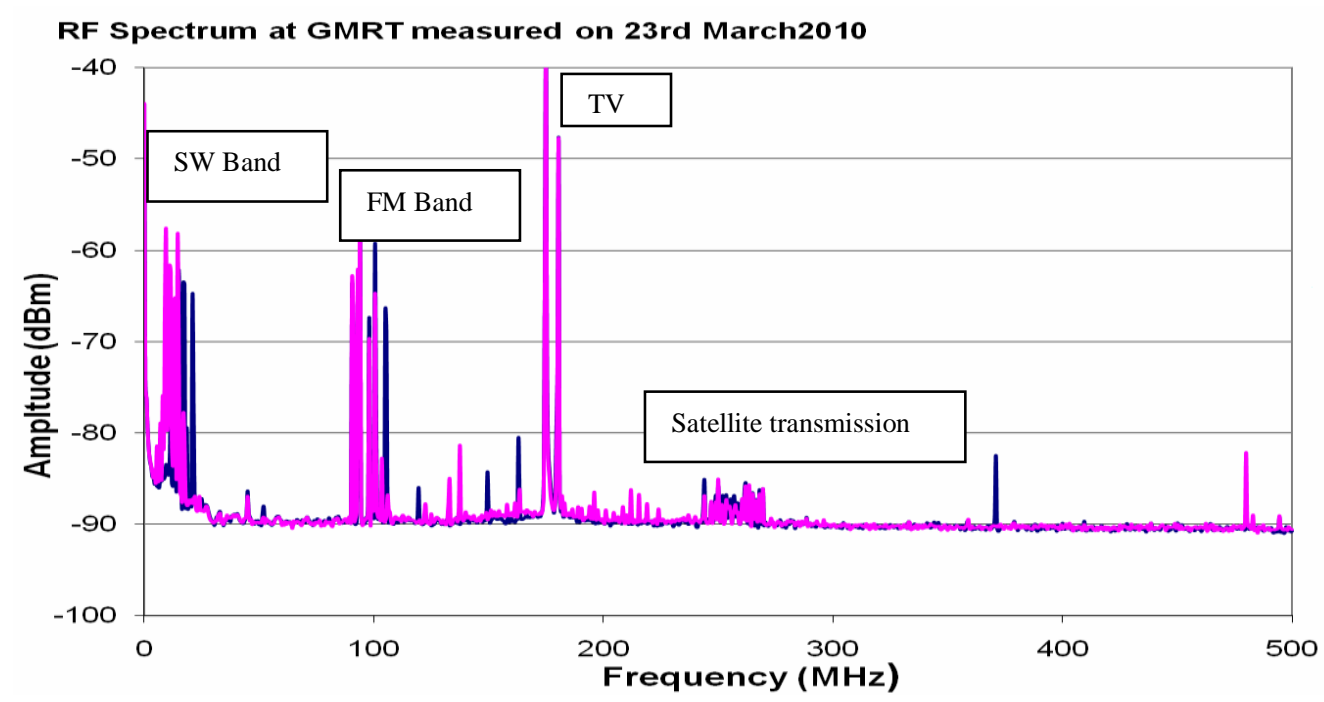

Figure 1.1a: RF Spectrum measured by the GMRT showing Short Wave communication transmission in the $9 \mathrm{KHz}-30 \mathrm{MHz}$ band, 88-108 MHz FM band, TV band CH-7 @ 174-180 $\mathrm{MHz}$, and satellite communications at 235-273 $\mathrm{MHz}$. 


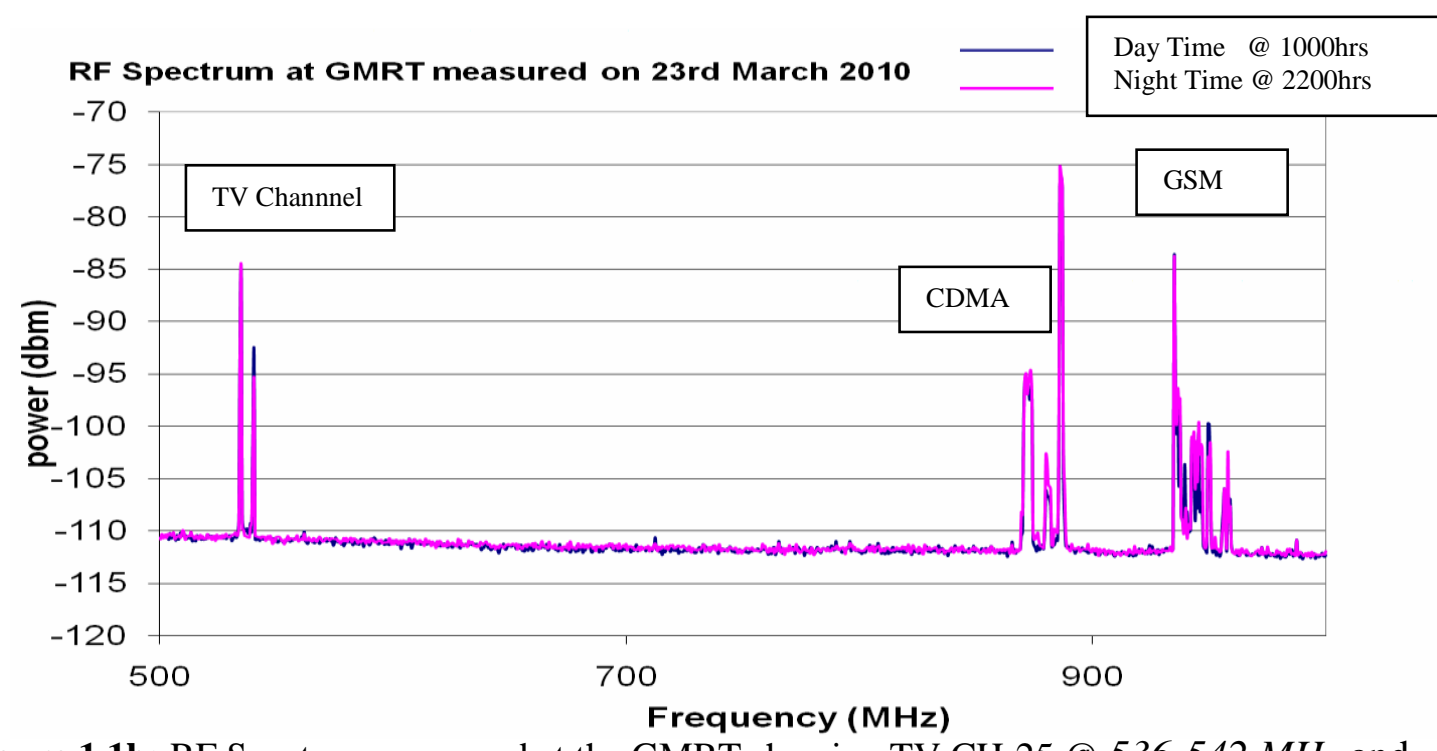

Figure 1.1b: RF Spectrum measured at the GMRT showing TV CH-25 @ 536-542 $\mathrm{MHz}$ and Mobile Frequencies covering CDMA 824-849 \& 869-894 MHz and GSM 896-901, \& 935-940 MHz bands.

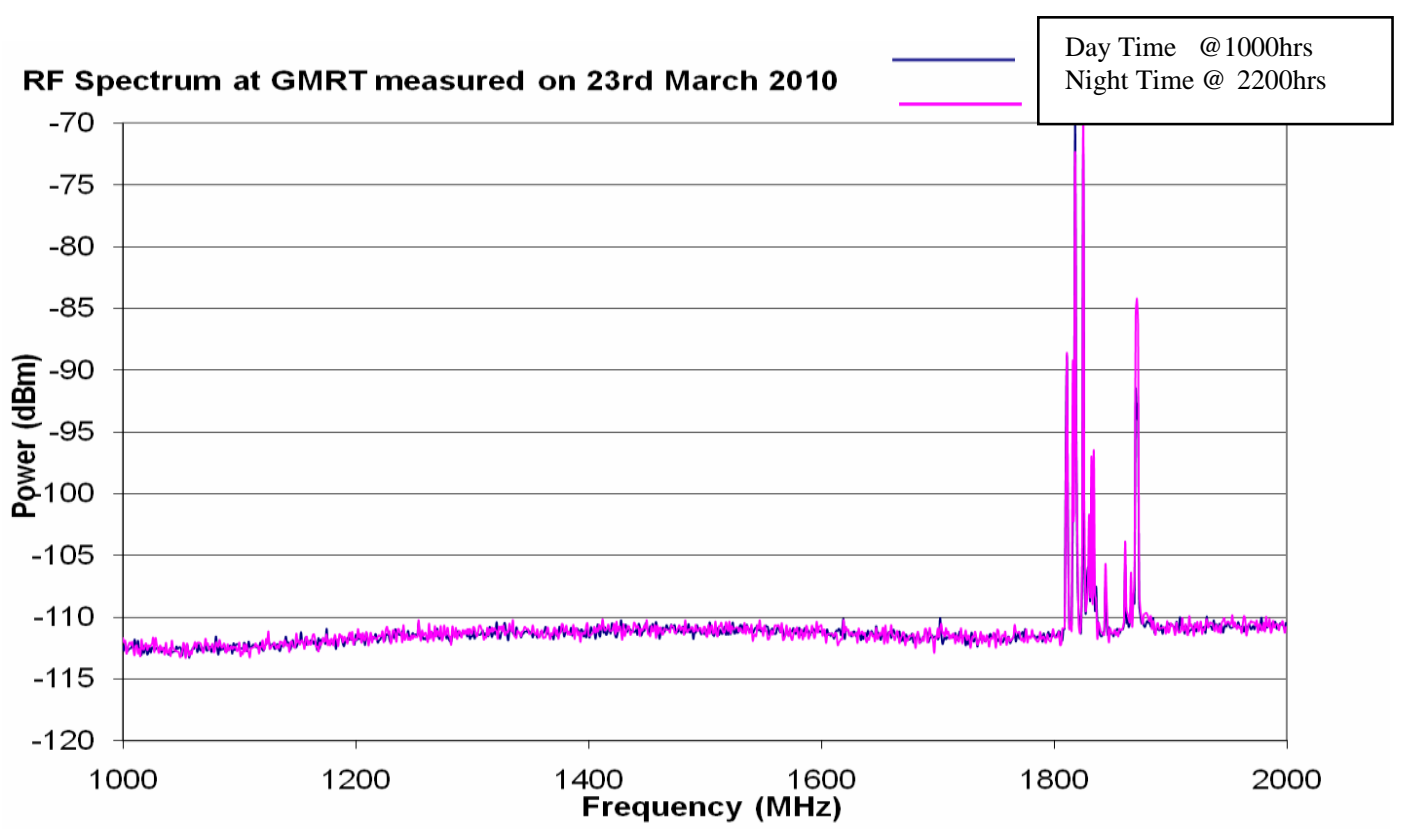

Figure 1.1c: RF Spectrum measured at the GMRT showing GSM mobile frequencies, 1850 $1910 \mathrm{MHz}$ band rest of the band no radiation is seen on the spectrum analyzer.

\subsection{Interference from TV boosters}

About 25 villages are located in and around GMRT. The GMRT array extends over a $14 \mathrm{~km}$ radial distance and is about $100 \mathrm{~km}$ from the nearest city, Pune, which has some TV transmitters. TV boosters are used by the village population to receive the weak signal from Pune. The TV boosters are of local make and are usually operated with very high gain, which saturates the booster amplifier and sometimes drives it into oscillation. About 25 villages were 
surveyed with support from the local administration. Figure 1.2a shows a typical signature of broadband and narrow band radiation from TV boosters producing interference in a GMRT band. Our RFI team has corrected the faulty TV boosters by limiting the gain of their saturated amplifiers by using a simple resistor, while ensuring good picture quality to the users. The modified TV boosters show no radiation as in figure $1.2 \mathrm{~b}$ shows. It took almost three years to complete the surveying and fixing of 1800 TV boosters. With advances in technology, about 66 $\%$ of their users have now switched to DTH services operating at Ku-band. This has also restricted the growth of cable TV networks in and around GMRT.

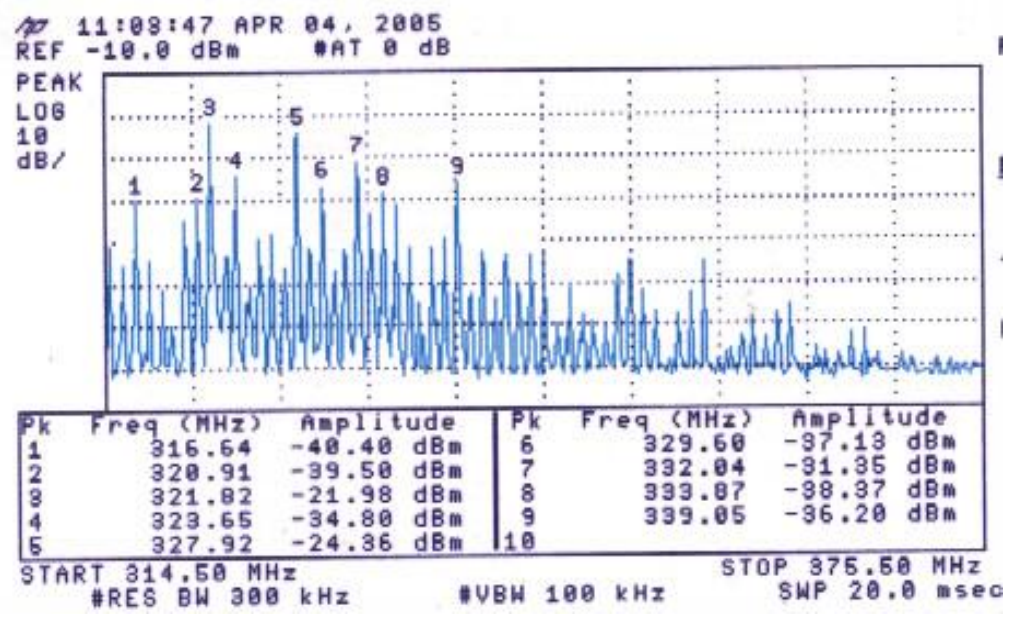

Figure 1.2a: RF spectrum from a radiating TV booster, Frequency $(314-375 \mathrm{MHz})$

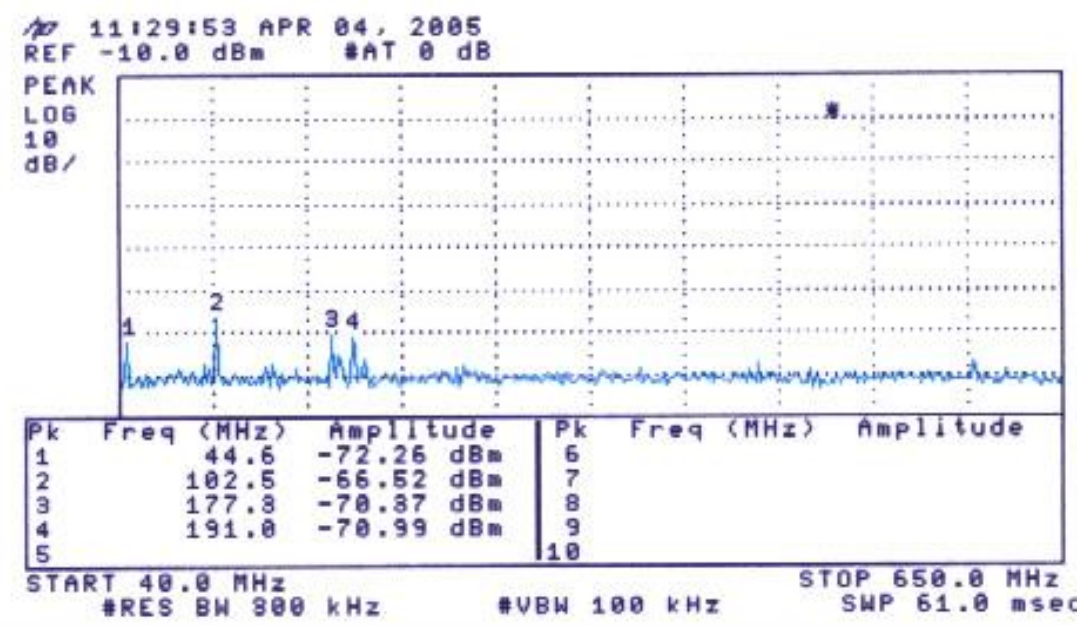

Figure 1.2b: RF spectrum after modification of the TV booster. 


\subsection{Interference from cell phone towers}

The GMRT L-band spectrum is divided into 4 sub-bands centered at $1060 \mathrm{MHz}, 1170 \mathrm{MHz}$, $1280 \mathrm{MHz}$ and $1390 \mathrm{MHz}$, each having a $110 \mathrm{MHz}$ bandwidth. The $1060 \mathrm{MHz}$ band is quite close to the $824-896 \mathrm{MHz}$ and $935-960 \mathrm{MHz}$ bands of the CDMA and GSM mobile operators respectively. With the growth of mobile phone operators in and around the GMRT, it was found that the $1060 \mathrm{MHz}$ band receivers go into saturation at low elevations, when on a line of site with a mobile tower. With the support of government agencies and good co-operation from the mobile operators, GMRT ensured $1800 \mathrm{MHz}$ band operation in and around the GMRT. In addition, these operators were asked to use low power transmitters and to defocus their antennae to avoid generating direct line of sight interference. Figures $1.3 \mathrm{a} \& 1.3 \mathrm{~b}$ show the spectrum around $950 \mathrm{MHz}$ before and after the shift by GSM to the $1800 \mathrm{MHz}$ band.

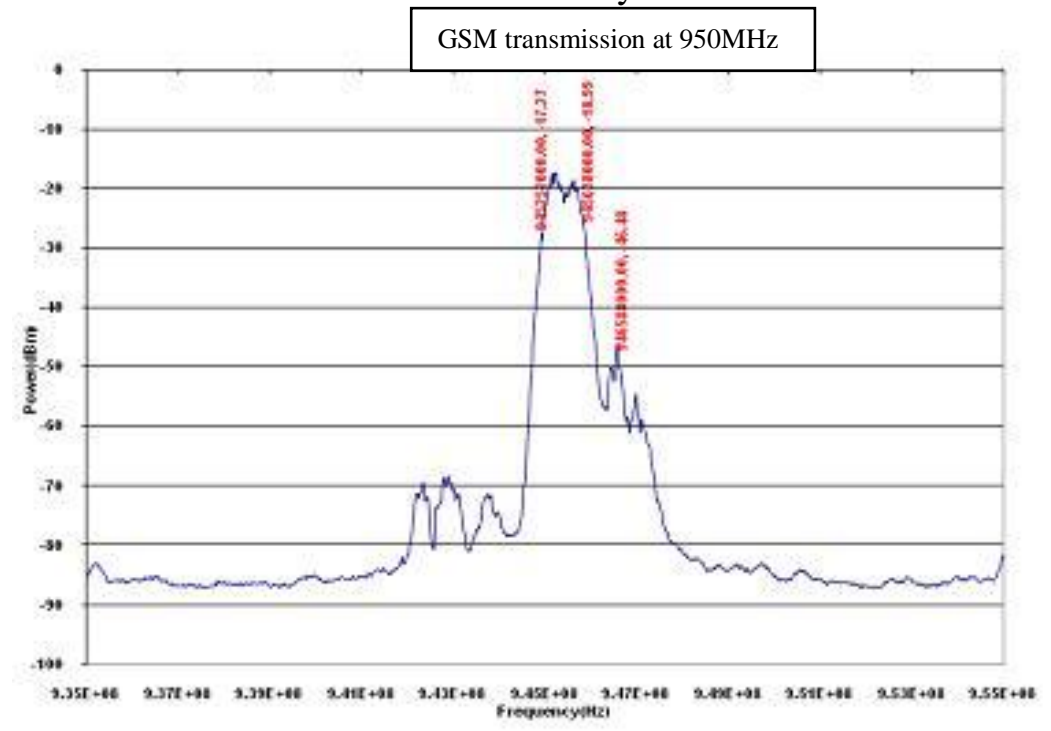

Figure 1.3a: RF spectrum in the GSM band @ 950 MHz showing very strong radiation

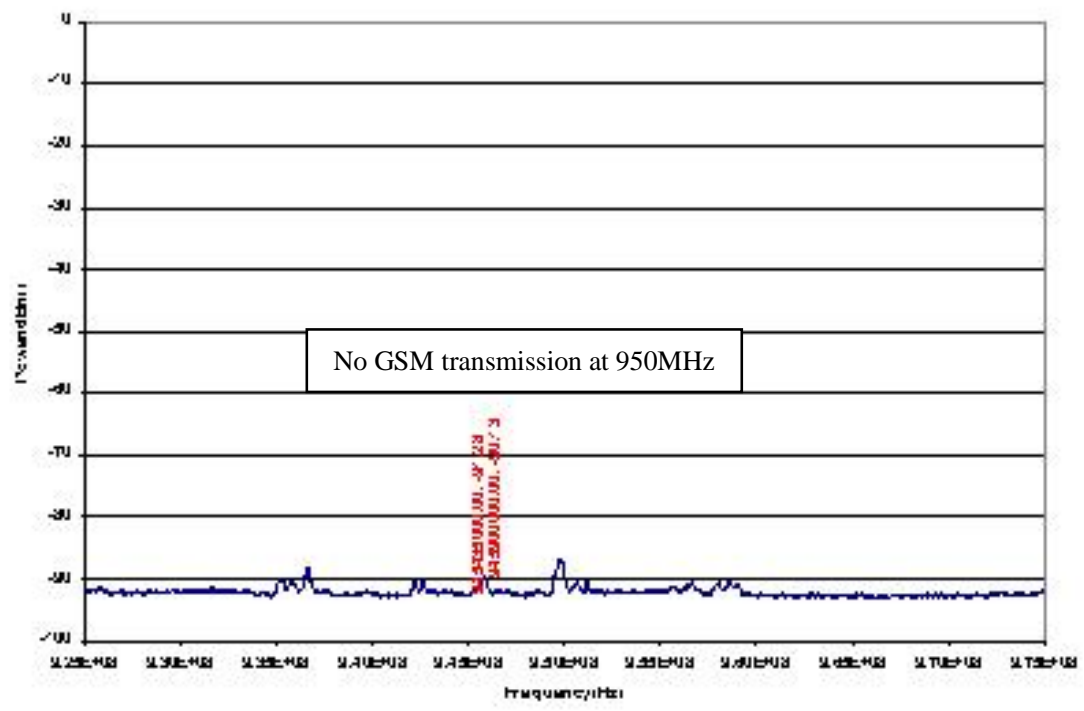

Figure 1.3b: RF spectrum in the GSM band @ $950 \mathrm{MHz}$ after the shift to $1800 \mathrm{MHz}$ operation, no transmissions are seen in the at $950 \mathrm{MHz}$ band. 


\subsection{Power line interference}

Power line interference is broadband in frequency and impulsive in time. The interference is due to arcing from (i) cracked or dirty insulators, (ii) badly cut joints on the over head lines, (iii) bad fuse links and fuse boxes at step down transformers, and (iv) poor earthing of electrical installations [1]. As the GMRT array extends over 25 villages, it encompasses a large number of domestic power lines that support residential and farming requirements, with both single and 3 phase transformers and distribution lines. In addition, a few high voltage transmission lines run near the outermost antennae of the GMRT Y array, with $11 \mathrm{KV}, 33 \mathrm{KV}, \mathrm{HVAC}$ and $500 \mathrm{KV}$ DC capacities.

Power line interference sources are located using an ultrasonic detector, which accurately pinpoints the sparking sources on the poles and on any other electrical installation. Using a log periodic antenna and spectrum analyzer, the detailed RF spectrum of the interference source is recorded and communicated to the electric company for rectification. As a regular practice, to keep power line interference under control, periodic surveys of electrical lines and installations are made to provide GPS locations for faulty equipment to aid in their rectification by the electric company. Figure 1.4a shows the location of all electrical installations in the western arm of the array: the classification of these faults is shown in figure 1.4b.

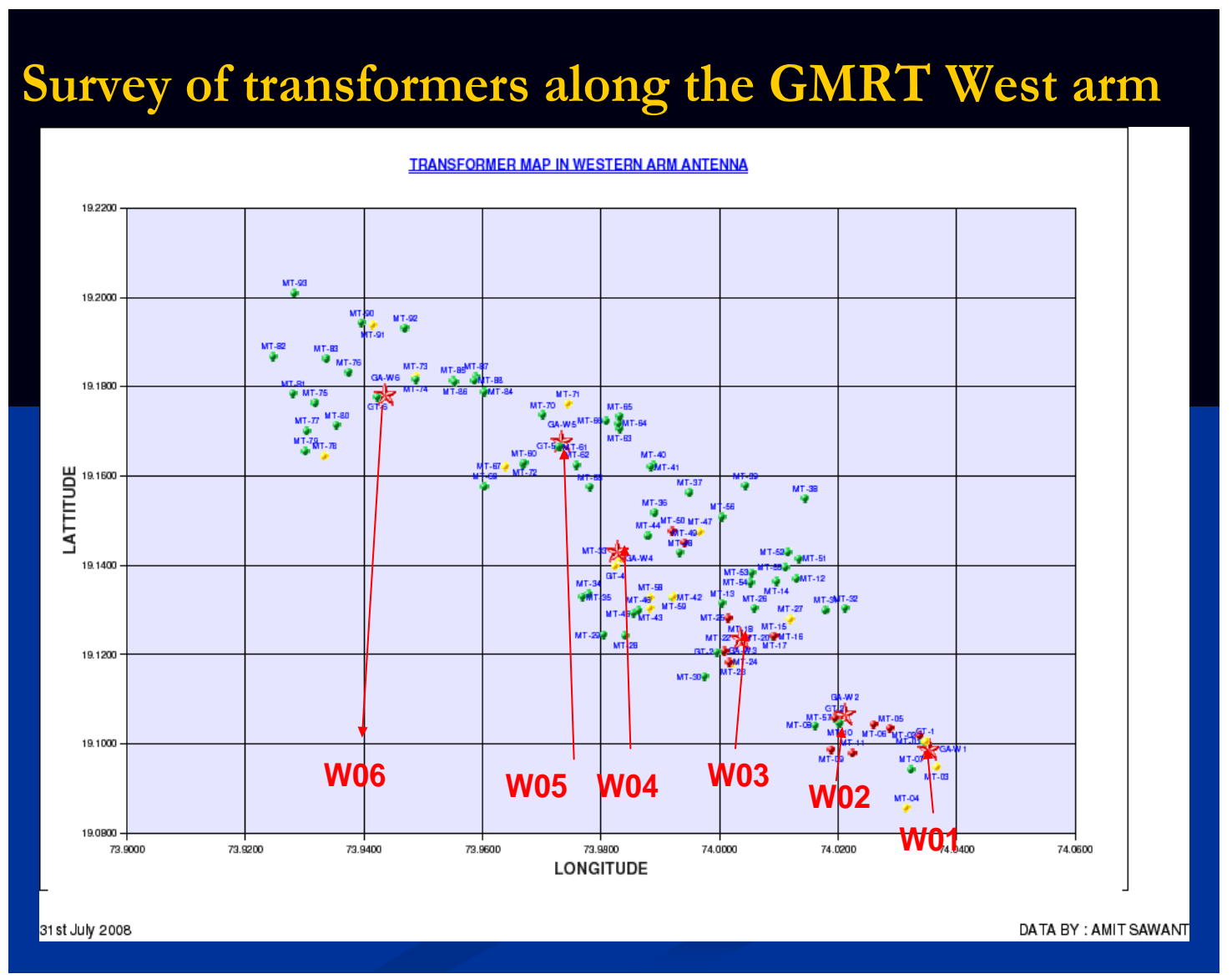

Figure 1.4a: Transformer installations in and around the western arm of the antenna site: a green dot at a good transformer, a red dot at a bad transformer and blue color text indicates transformer number. 


\begin{tabular}{|c|c|c|c|c|c|}
\hline SR. & ANTENNAS & TRANSFORMERS & PROBLEMATIC & AVERAGE & GOOD \\
\hline NO. & & ATTENDED & & & \\
\hline 1 & EAST ARM & 5 & E-02,E-05 & & E-03,E-04,E-06 \\
\hline 2 & WEST ARM & 6 & W-01,W-03 & & W-02,W-04,W-05,W-06 \\
\hline 3 & SOUTH ARM & 5 & S-01 & & S-02,S-03,S-04,S-06 \\
\hline & TOTAL & 16 & 5 & & 11 \\
\hline SR. NO. & \multicolumn{2}{|c|}{ DETAILS OF RADIATION } & EAST & WEST & SOUTH \\
\hline 1 & \multicolumn{2}{|c|}{ BAD 11KV DISC INSULATOR } & & W-01 & \\
\hline 2 & \multicolumn{2}{|l|}{ BAD DO } & E-02 & W-01 & \\
\hline 3 & \multicolumn{2}{|c|}{ BAD TRANSFORMER } & E-02,E-05 & W-01, W03 & S-01 \\
\hline 4 & \multicolumn{2}{|c|}{ BAD H.T. BUSHING PIN INSULATOR } & & W-01 & \\
\hline 5 & \multicolumn{2}{|c|}{ BAD L.T. BUSHING PIN INSULATOR } & & W-01 & \\
\hline
\end{tabular}

Figure 1.4b: Details of the problem at transformer installation sites along the eastern, southern and western arms of the GMRT antenna.

\subsection{RFI detection using the GMRT software backend (GSB)}

The recently developed GMRT software backend (GSB) [2] uses a high-performance computing platform assembled from off-the-shelf commodity machines with minimal hardware investment. This gives flexibility in final time and frequency resolution, and an ability to implement algorithms for radio frequency interference rejection. The GSB backend was also developed for using the GMRT to study the Epoch of Reionisation by Ue-Li Pen [3] and his collaborators from the GMRT, who have used it to locate the strong sources of RFI using interferometric techniques.

This approach was used to locate a hanging stray wire on a $440 \mathrm{KV}$ AC EHT line, which was generating the broadband RFI shown in figure 1.5. The hanging stray wire has been removed by the electric company using isolating rods. Similarly, an abandoned telephone pole with hanging wire near a high-tension line was producing RFI that was located using the software backend: and the pole was removed thus reducing interference from the location. 


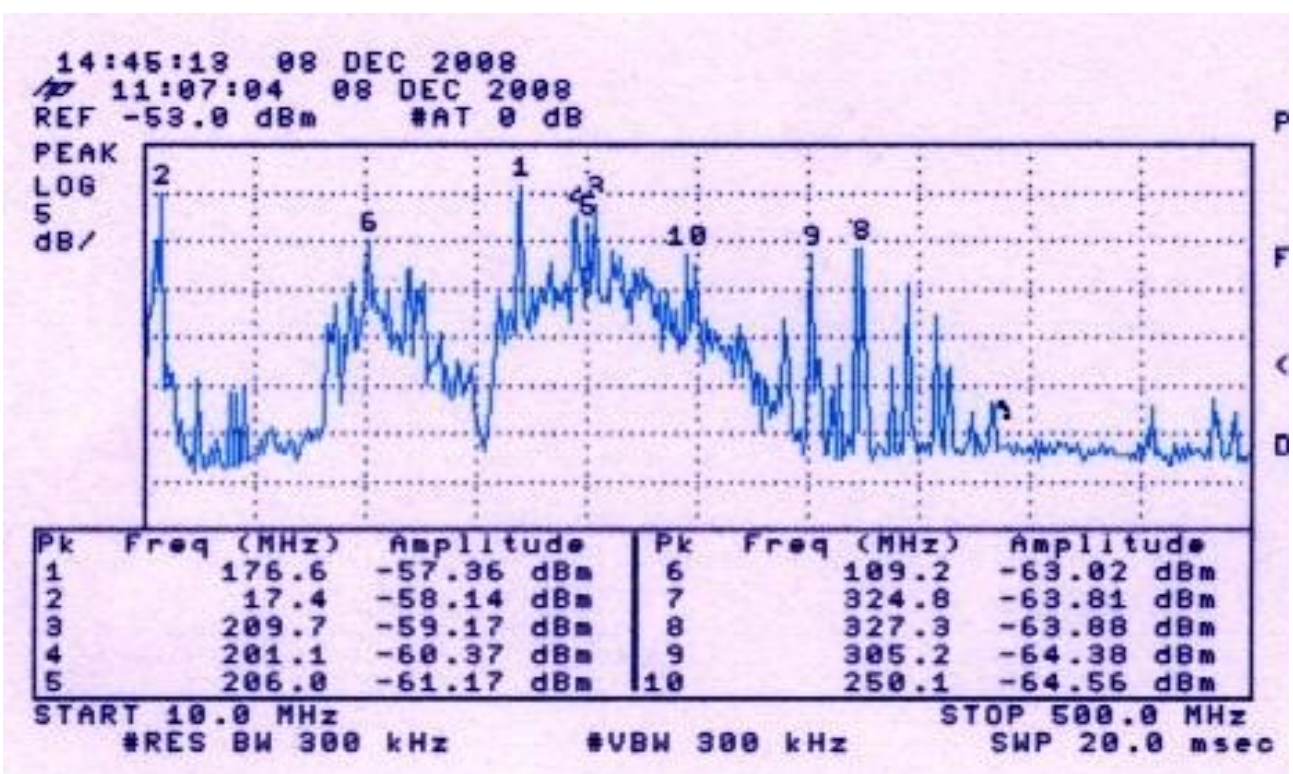

Fig.1.5: Power line interference measured on a spectrum analyzer using a log-periodic antenna, covering the $10-400 \mathrm{MHz}$ frequency range.

\subsection{Interference from industrial areas.}

With the help of government agencies, the GMRT could ensured an industry-free zone over the entire GMRT array. Industries built well-away from the GMRT array were also asked to secure approval from the GMRT to ensure that they do not generate any radio frequency interference to the GMRT. The GMRT ensures that no RFI-producing DC machines are used by these industries, and also ensures that proper electrical wiring and installation is performed before giving approval for an industry.

\subsection{Conclusions}

The GMRT is located in a region of high population density, and must hence co-exist with multiple sources of RFI.

The primary sources of RFI at the GMRT are from cellular phone transmissions, malfunctioning power lines, equipment and TV boosters.

Regular field surveys are carried out to locate sources of strong RFI. Direct repairs of individual TVs were used to solve the problem of malfunctioning TV boosters. New analysis techniques using the fully real-time software backend (GSB) give the ability to implement algorithms for radio frequency interference rejection and to locate sources of RFI using interferometric techniques.

\section{References}

[1] Edward N. Skomal, Man-Made Radio Noise, VNB publications, 1978.

[2] Jayanta Roy, Yashwant Gupta, Ue-Li Pen, Jeffrey B. Peterson, Jitendra Kodilakar, Sanjay Kudale, A real time software backend for the GMRT. 2010

[3] Pen et al 2009 